\title{
4D Quantitative Coronary Artery Motion Analysis: A Novel Method for Culprit Lesion Prediction
}

\author{
Benjamin Griffiths ${ }^{1, *}$, Jean-Paul Aben ${ }^{2}$, Aiden O'Loughlin ${ }^{1}$ \\ ${ }^{1}$ School of Medicine, Western Sydney University, Campbelltown, Sydney, NSW, 2560, Australia \\ ${ }^{2}$ Pie Medical Imaging, the Netherlands
}

Copyright $\mathrm{C} 2018$ by authors, all rights reserved. Authors agree that this article remains permanently open access under the terms of the Creative Commons Attribution License 4.0 International License

\begin{abstract}
This study aims to determine if measuring four-dimensional quantitative coronary artery motion (QCAM) and change in tortuosity $(\Delta \mathrm{T})$ on invasive biplane coronary angiogram is predictive of the location of culprit lesions responsible for myocardial infarctions. Invasive coronary angiograms have no current clinical application for the prediction of future coronary events. Previous studies have shown promise in demonstrating the effects of coronary artery motion on plaque formation and location, but this has yet to fully translate into a directed diagnostic method. QCAM and $\triangle T$ were calculated with CAAS QCA4D prototype software (Pie Medical Imaging, the Netherlands) for sections of the culprit coronary artery using biplane coronary angiograms of fourteen patients undergoing percutaneous coronary intervention for myocardial infarction. Prediction of the artery section containing the culprit lesion was performed using one sample t-testing, generalised linear latent and mixed statistical modelling with grouping by patient, and logistic regression modelling. QCAM was a significant predictor of the location of culprit lesions $(p=0.047) . \Delta T$ was not a significant predictor of the location of culprit lesions $(p=0.49)$. QCAM has a role in predicting the location of culprit lesions, and may allow for targeted local therapy to prevent future events.
\end{abstract}

Keywords Coronary Artery Motion, Coronary Physiology, Coronary Angiography, Myocardial Infarction

\section{Introduction}

Coronary artery disease (CAD) is a leading cause of morbidity and mortality worldwide. It accounted for 7.4 million deaths globally in 2013 [1], when it was also the leading single cause of death in the US [2]. Approximately every 40 seconds, an American will have a heart attack [3]. Direct and indirect costs of CAD totaled over USD \$199 billion in the US for 2012-13 [3]. Global disability-adjusted life years (DALYs) from CAD, representing health years of life lost, are expected to rise from 47 million in 1990 to 82 million in 2020 [4]. CAD demonstrates an ongoing challenge for medical professionals and the public health sector alike in its burden and ubiquity.

Myocardial infarction, a result of CAD, most commonly occurs with the rupture or erosion of atherosclerotic plaque and subsequent occlusive thrombus formation. These plaques form in response to endothelial injury [5], such as caused by hypertension, smoking and dyslipidaemia, in a non-uniform manner throughout the coronary vasculature [6]. Their distribution can likely be attributed to local factors relating to fluid dynamics and wall mechanics. Coronary artery motion (CAM) is one such established local biomechanical factor [7]. Despite the relative dominance of the 'shear stress' model of plaque-causing injury in the literature, CAM is emerging as a promising new avenue for expanding the knowledge base surrounding atherogenesis and developing novel predictive imaging technologies.

Qualitative evaluation of coronary artery motion has previously demonstrated the compression type of motion to correlate with the location of disease and the degree of stenosis within coronary artery segments [8] and is independently predictive of the location of future culprit lesions responsible for ST segment elevation myocardial infarctions $[9,10]$.

The development of a quantitative method, using either multislice computed tomography (CT) coronary angiography or invasive coronary angiography, has furthered our understanding of the role of CAM. A significant correlation was found between a quantitative index of vessel centreline shortening and the location of coronary plaque and the degree of stenosis in patients having CT coronary angiography [11]. A quantitative index of the compression type of coronary artery motion obtained from analysis of invasive coronary angiograms has previously been shown to be predictive of the location of future culprit lesions responsible for ST segment 
elevation myocardial infarctions [12]. These findings indicate the potential for a quantifiable unit of artery motion, obtained from coronary imaging, to have clinical use in characterizing patient's coronary vasculature and predicting coronary events.

Limited research has been conducted into the role of tortuosity in the pathogenesis of coronary artery disease, although it has been shown that severe tortuosity is linked with lower incidence of significant disease [13]. The significance of this, however, remains unknown.

Hence, this study aims to see if a quantitative index of the compression type of coronary artery motion (QCAM) and change in tortuosity $(\Delta \mathrm{T})$ calculated with CAAS QCA4D prototype software (Cardiovascular Angiographic Analysis Systems Quantitative Coronary Analysis in 4D) (Pie Medical Imaging, the Netherlands) predicts the location of culprit lesions responsible for myocardial infarctions.

\section{Methods}

\section{Patients}

Patients were identified using the angiography database at Westmead Hospital in Sydney who had biplane coronary angiography and percutaneous coronary intervention performed for acute myocardial infarction.

Experienced interventional cardiologists performed the angiography, identified the culprit lesion, and then proceeded to treat the culprit lesion by stenting. Patients were excluded if they had previous coronary artery bypass surgery, stent thrombosis, or in-stent or in-segment restenosis.

\section{Calculation of QCAM}

The biplane angiograms of the coronary arteries containing the culprit lesion were opened in the CAAS QCA4D prototype software (Pie Medical Imaging, the Netherlands) program. Each artery had its main vessel divided into sections by identifying corresponding section start and end locations in both angiographic views. A typical artery would have the first section from the ostium to a major anatomical landmark (usually the origin of a major branch), with a second and subsequent sections from the end of the preceding section to the next major anatomical landmark that was identifiable in both angiographic views. End-diastole was identified as the largest cardiac silhouette within the cardiac cycle close to the peak of the R-wave in the ECG, and end-systole was identified as the smallest silhouette close to the end of the T-wave. Sequentially, for each individual frame of a full cardiac cycle (from end-diastole to the next end-diastole), the 'normal' 3D model was generated with the software [14] for each coronary section. Figure 1 shows an example of a 3D model.

After creation of a three-dimensional model, the software was used to calculate the centreline length and tortuosity of each section for that frame of the cardiac cycle. QCAM and change in tortuosity $(\Delta \mathrm{T})$ were derived from these measurements. QCAM was defined as the ratio of the section lengths at end-diastole and end-systole represented as a percentage i.e. QCAM $=100 *$ (systolic length/diastolic length). A QCAM value of 100 represented no change in length of the section from end-diastole (ED) to end-systole (ES), with greater and lesser values corresponding to section lengthening and shortening respectively.

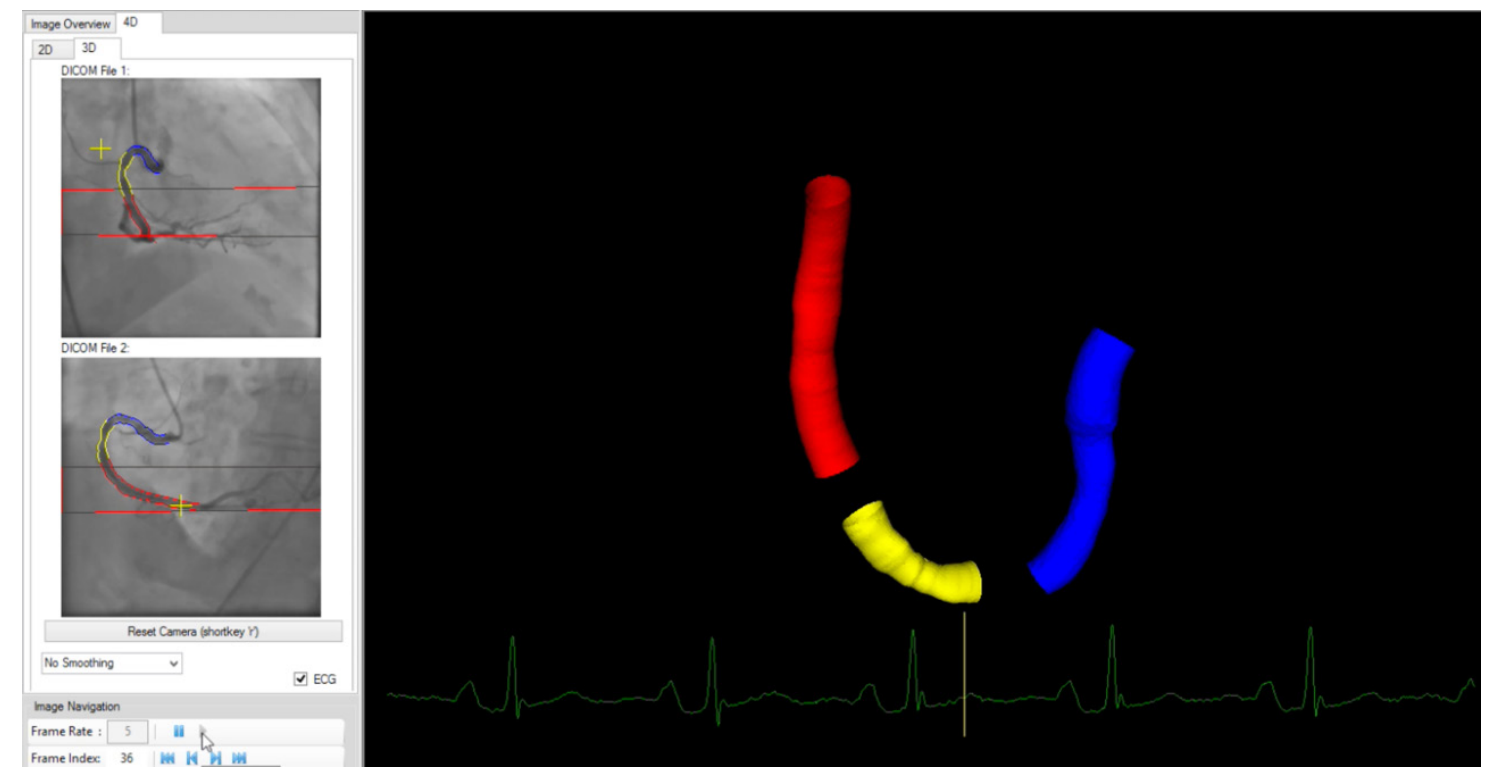

Figure 1. A three-dimensional model created with CAAS QCA4D prototype software (Pie Medical Imaging, the Netherlands) 


\section{Calculation of Tortuosity}

The tortuosity measure gives information about how curved (i.e. tortuous) a vessel is, and is calculated based on the amount and amplitude of bends. A 3D centerline, depicting the vessel as it would appear without torsion, was constructed by creating a polyline with vertices at bending points (positions of minimum curvature) in the vessel. The tortuosity measure equals the sum of the actual length of each section divided by the reconstructed polyline length minus one.

Change in tortuosity $(\Delta \mathrm{T})$ was the difference between the tortuosity measurements for the section at end-diastole and at end-systole i.e. $\Delta \mathrm{T}=\mathrm{T}_{\text {End-diastole }}-\mathrm{T}_{\text {End-systole. }}$.

\section{Statistical Analysis}

Prediction of an artery section containing the culprit lesion was performed using one-sample t-testing, a generalised linear latent and mixed statistical model with grouping by patient, and a logistic regression model. Statistical analysis was performed using STATA version 10 (StataCorp LP. College Station, TX, USA). A p value $<0.05$ was considered significant.

\section{Ethics Approval of the Study Protocol}

The human research ethics committee of Royal Prince Alfred Hospital approved the study (Protocol No X10-0159 \& HREC/10/RPAH/291). This approval was recognized by the Western Sydney University HREC (UWS H11293).

\section{Results}

Fourteen patients were identified and their biplane angiograms analysed. The patients had their procedures between December 2011 and April 2014. Table 1 shows patient demographics and culprit artery distribution. The culprit lesions causing non-STEMI were present in six left anterior descending coronary, seven right coronary and one left circumflex coronary arteries. There were five STEMI and nine non-STEMIs.

Table 1. Demographics of patients and culprit artery distribution

\begin{tabular}{|c|c|}
\hline Age (years) $+/$ - standard deviation & $56+/-9$ \\
\hline Male gender (\%) & 80 \\
\hline Culprit artery & \\
\hline Left anterior descending & 6 \\
\hline Right & 7 \\
\hline Left Circumflex & 1 \\
\hline Type of Myocardial Infarction & 5 \\
\hline STEMI & 9 \\
\hline Non-STEMI & \\
\hline
\end{tabular}

Five hundred and fifty one three-dimensional reconstructions were made of thirty nine sections representing an average of 14 reconstructions to complete a full cardiac cycle for each artery section. The mean QCAM across all non-culprit sections was $95.33 \%(+/-3.08$ (standard error of the mean (SEM))). The mean QCAM across all culprit sections was $81.59 \%(+/-6.25$ (SEM)). These results are shown in Table 2. A one sample t-test comparing QCAM for the non-culprit and culprit sections was statistically significant with a p-value of 0.047 .

The mean $\Delta \mathrm{T}$ across all non-culprit sections was 0.0083 . $(+/-3.08$ (standard error of the mean (SEM))). The mean $\Delta \mathrm{T}$ across all culprit sections was $0.0056(+/-6.25$ (SEM)). These results are shown in Table 3. A one sample t-test comparing $\Delta \mathrm{T}$ for the non-culprit and culprit sections was not statistically significant with a p-value of 0.16 .

Figure 2 is a frequency histogram of QCAM grouped by non-culprit and culprit sections. Non-culprit values ranged from 74.68 to 141.82 whilst culprit lesions ranged from 34.02 to 109.33 .

Figure 3 shows the QCAM values for each artery sections for each individual patient.

A generalized linear mixed model with explanatory variables of QCAM and $\triangle \mathrm{T}$ and grouping by patient was used to assess the outcome of an artery section being the culprit. QCAM was predictive of the culprit artery section $(\mathrm{p}=0.047) . \Delta \mathrm{T}$ was not predictive $(\mathrm{p}=0.49)$.

A logistic regression model with QCAM as an explanatory variable and culprit section as the outcome variable was created. This was used to evaluate the value of QCAM for predicting the probability of a section being a culprit, as shown in Figure 4.

Table 2. Summary statistics for QCAM by non-culprit and culprit section types

\begin{tabular}{|c|c|c|c|c|}
\hline $\begin{array}{c}\text { Section } \\
\text { Type }\end{array}$ & Frequency & Mean & $\begin{array}{c}\text { Standard Error } \\
\text { of the Mean }\end{array}$ & $\begin{array}{c}\text { Standard } \\
\text { Deviation }\end{array}$ \\
\hline Non- culprit & 25 & 95.33 & 3.08 & 15.41 \\
\hline Culprit & 14 & 81.59 & 6.25 & 23.40 \\
\hline
\end{tabular}

Table 3. Summary statistics for $\Delta \mathrm{T}$ by non-culprit and culprit section types

\begin{tabular}{|c|c|c|c|c|}
\hline $\begin{array}{c}\text { Section } \\
\text { Type }\end{array}$ & Frequency & Mean & $\begin{array}{c}\text { Standard Error } \\
\text { of the Mean }\end{array}$ & $\begin{array}{c}\text { Standard } \\
\text { Deviation }\end{array}$ \\
\hline $\begin{array}{c}\text { Non- } \\
\text { culprit }\end{array}$ & 25 & 0.0083 & 0.0030 & 0.0149 \\
\hline Culprit & 14 & 0.0056 & 0.0018 & 0.0069 \\
\hline
\end{tabular}

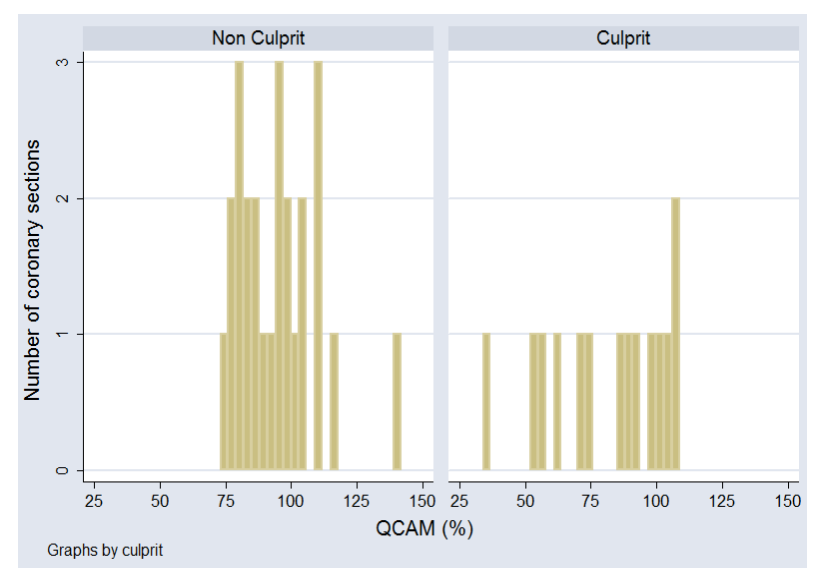

Figure 2. Frequency histogram of QCAM grouped by non-culprit and culprit sections 


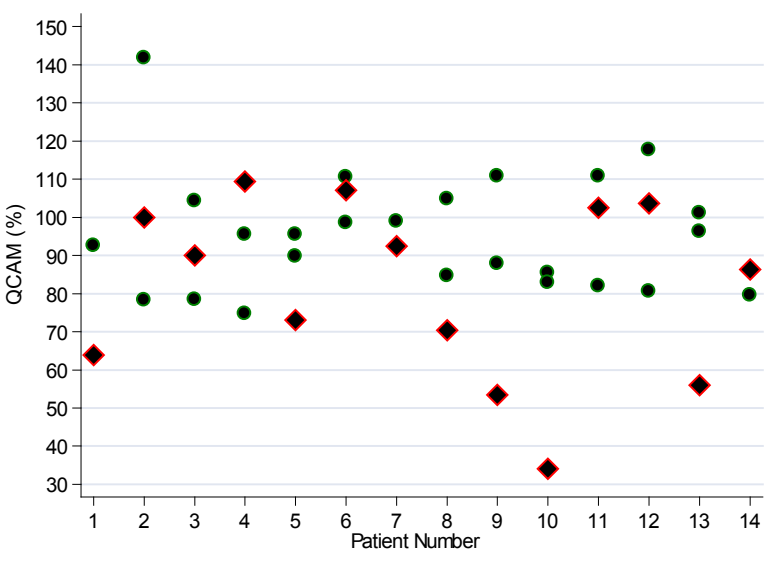

Figure 3. QCAM for non-culprit (green) and culprit (red) sections for each individual patient

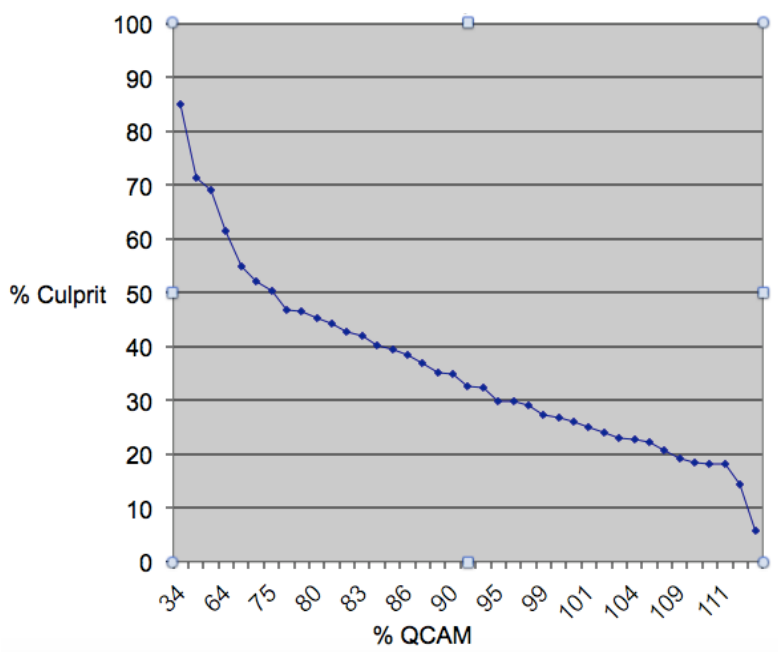

Figure 4. Prediction using logistic model with QCAM as a single explanatory variable

\section{Discussion}

These results demonstrate a statistically significant association between QCAM and the location of culprit lesions responsible for myocardial infarctions. This is likely explained by coronary artery motion acting as a local biomechanical factor of endothelial injury. QCAM quantifies the mechanical stress exerted by the stretch-compression forces exerted upon a vessel during the cardiac cycle. As a result, this may allow for prediction of atherogenesis and plaque rupture.

This association may also play a role in the mechanism by which unusual psychological stress, hypertension and a rise in catecholamine levels have been shown to be triggers for myocardial infarctions [15-19]. In animal studies, administration of vasopressors significantly increased coronary artery motion parameters, such as distance moved, maximum velocity, acceleration and decelerations [20]. These changes demonstrate how QCAM, in combination with previously recognised instigators of plaque destabilization such as high wall stress and shear-stress-induced weakening [21], are involved in the pathogenesis of acute thrombus formation.

Figure 2 shows that the distribution of QCAM values for culprit and non-culprit sections have considerable overlap, despite their significantly differing mean values. One possible explanation for this is that there are non-culprit lesions under the same biomechanical stress as established culprit lesions that may too develop into culprit lesions in the future. These sections may be influenced by local protective factors, such as high shear stress and low strain, preventing abnormal blood flow and atherogenesis. Alternatively, this overlap may be due to measurement error, and improvements in measuring the biomechanical stress of coronary artery motion may reveal a truer QCAM range for culprit and non-culprit lesions.

There is no current direct clinical application for this research. The software used currently requires input from angiography performed with a biplane system, rather than the more commonly used monoplane angiography systems. Further development of this line of research does, however, lead to a number of conceivable future clinical applications. Better understanding of the biomechanical effects of current generation stent technologies may lead to stent strategies that result in lower rates of major adverse cardiac events. Previous reports have shown that longitudinal straightening effect of stents to be a statistically significant predictor of major adverse cardiac events [22]. Modelling of a double stent versus a single stent strategy for a curved artery has suggested higher flexibility, more conformity and lower recoil [23]. Whether the interaction of different stent strategies and local biomechanical stress in stented segments will result in different clinical outcomes is an untested hypothesis and a suggestion for future study.

Bioresorbable vascular scaffolds, which have been termed the 'fourth revolution' in interventional cardiology [24], have been approved for a number of current stent indications. They are also being trialed for the novel indication of treatment of non-obstructive vulnerable plaques, isolated cases of this use having already been reported in the literature $[25,26]$. The ABSORB Bioresorbable Vascular Scaffold is being used for this indication in the randomized multicentre Scandinavian PROSPECT ABSORB substudy of the PROSPECT II trial (which aims to enroll 900 patients). A favorable result in this study would highlight the importance of novel methods, such as QCAM, for predicting the location of future culprit plaques.

One limitation of this study was the software's prototypical nature, which led to some difficulties in tracing artery segments, namely with vessels that ran parallel to the line of sight. It also requires input from the less commonly used biplane modality, restricting recruitment and resulting in the small sample size. Future studies should endeavor to have a larger sample size to confirm these findings. The study was also limited by an $80 \%$ male cohort. 
Another recommendation for future research is to explore the relationship between left ventricular ejection fraction (LVEF), coronary artery motion and compression and tortuosity measurements, and consideration for analysis of interaction. LVEF is associated with cardiac wall motion [27] and hence coronary haemodynamics [28].

\section{Conclusions}

This study identifies that QCAM has a role in predicting the location of culprit lesions. This finding proposes that the biomechanics of coronary artery motion play a significant role in plaque formation and rupture, and suggests an alternative mechanism of injury to the predominating theories of abnormal wall and shear stress. QCAM provides a clinically accessible method to predict the location of culprit lesions responsible for myocardial infarctions and may direct novel strategies to help prevent these events.

\section{REFERENCES}

[1] World Health Organisation. Global status report on noncommunicable diseases 2014. Geneva, Switzerland; 2014. Available from:

http://apps.who.int/iris/bitstream/10665/148114/1/9789241 564854_eng.pdf?ua $=1$

[2] World Health Organisation. Global health estimates 2015: deaths by cause, age, sex, by country and by region, 2000-2015. Geneva, Switzerland; 2016. Available from: http://www.who.int/healthinfo/global_burden_disease/estim ates/en/index1.html

[3] Benjamin E, Blaha M, Chiuve S, Das S, Deo R et al. Heart disease and stroke statistics - 2017 update: a report from the American Heart Association. Circulation. 2017 Apr $18 ; 135(16)$.

[4] World Health Organisation. Global atlas on cardiovascular prevention and control. Geneva, Switzerland; 2011. Available from:

http://www.who.int/cardiovascular_diseases/publications/at las_cvd/en/

[5] Davignon J, Ganz P. Role of endothelial dysfunction in atherosclerosis. Circulation. 2004 Jun 15;109:27-32.

[6] Cheruvu P, Finn A, Gardner C, Caplan J, Goldstein J et al. Frequency and distribution of thin-Cap fibroatheroma and ruptured plaques in human coronary arteries: a pathologic study. J Am Coll Cardiol. 2007 Sep 4;50(1):940-9.

[7] John LC. Biomechanics of coronary artery and bypass graft disease: potential new approaches. Ann Thorac Surg. 2009 Jan;87(1):331-8.

[8] Konta T, Bett JH. Patterns of coronary artery movement and the development of coronary atherosclerosis. Circ J. 2003 Oct;67(10):846-50.
[9] Chan KH, Chawantanpipat C, Gattorna T, Chantadansuwan T, Kirby A, Madden A, et al. The relationship between coronary stenosis severity and compression type coronary artery movement in acute myocardial infarction. Am Heart J. 2010 Apr;159(4):584-92.

[10] O'Loughlin AJ, Byth K. The stretch-compression type of coronary artery movement predicts the location of culprit lesions responsible for ST-segment elevation myocardial infarctions. Heart Lung Circ. 2007 Aug;16(4):265-8.

[11] O'Loughlin AJ, Tang L, Moses D, Tong W, French JK, Richards DA, et al. A Novel Quantitative Index of Coronary Artery Motion from Multislice Computed Tomography and the Location of Coronary Artery Disease. International Journal of Cardiovascular and Cerebrovascular Disease. 2014;2(1):1-5.

[12] O'Loughlin A, Kazi S, French JK, Richards DA, Denniss AR, Hennessy A. Quantitative Coronary Artery Motion Analysis Predicts the Location of Future ST Segment Elevation Myocardial Infarctions. International Journal of Cardiovascular and Cerebrovascular Disease. 2014;2(3):35-8.

[13] Groves SS, Jain AC, Warden BE, Gharib W, Beto RJ 2nd. Severe coronary tortuosity and the relationship to significant coronary artery disease. W V Med J. 2009 Jul-Aug;105(4):14-7.

[14] Onuma Y, Girasis C, Aben JP, Sarno G, Piazza N et al. A novel dedicated 3-dimensional quantitative coronary analysis methodology for bifurcation lesions. EuroIntervention. 2011 Sep 30;7(5):629-35.

[15] Fuster V, Badimon L, Badimon JJ, Chesebro JH. The pathogenesis of coronary artery disease and the acute coronary syndromes (2). N Engl J Med. 1992 Jan 30;326(5):310-8.

[16] Leor J, Poole WK, Kloner RA. Sudden cardiac death triggered by an earthquake. N Engl J Med. 1996 Feb 15;334(7):413-9.

[17] Fuster V, Badimon L, Badimon JJ, Chesebro JH. The pathogenesis of coronary artery disease and the acute coronary syndromes (1). N Engl J Med. 1992 Jan. $23 ; 326(4): 242-50$

[18] Kales SN, Soteriades ES, Christophi CA, Christiani DC. Emergency duties and deaths from heart disease among firefighters in the United States. N Engl J Med. 2007 Mar 22;356(12):1207-15.

[19] Wilbert-Lampen U, Leistner D, Greven S, Pohl T, Sper S, Volker C, et al. Cardiovascular events during World Cup soccer. N Engl J Med. 2008 Jan 31;358(5):475-83.

[20] Kurosawa H, Seto Y, Wakamatsu H, Sato Y, Takase S, Omata $\mathrm{S}$, et al. Effects of phenylephrine and noradrenaline on coronary artery motion in an open-chest porcine beating heart model. Surg Today. 2014 Jun;44(6):1128-38

[21] Kwak B, Bäck M, Bochaton-Piallat M-L, Caligiuri G, Daemen $\mathrm{M}$ et al. Biomechanical factors in atherosclerosis: mechanisms and clinical implications. Eur Heart J. 2014 Nov 14;35(43):3013-20.

[22] Gyöngyösi M, Yang P, Khorsand A, Glogar D. Longitudinal straightening effect of stents is an additional predictor for 
major adverse cardiac events. J Am Coll Cardiology. 2000;35(6):1580-9.

[23] Kasiri S, Kelly DJ. An argument for the Use of multiple segment stents in curved arteries. J Biomech Eng. 2011 Sep $15 ; 133(8)$.

[24] Serruys PW, Garcia-Garcia HM, Onuma Y. From metallic cages to transient bioresorbable scaffolds: change in paradigm of coronary revascularization in the upcoming decade? Eur Heart J. 2012;33(1):16-25.

[25] Gomez-Lara J, Garcia-Garcia HM, Onuma Y, Garg S, Regar E, De Bruyne B, et al. A Comparison of the Conformability of Everolimus-Eluting Bioresorbable Vascular Scaffolds to
Metal Platform Coronary Stents. JACC: Cardiovascular Interventions. 2010;3(11):1190-8.

[26] Ramcharitar S, Gonzalo N, van Geuns RJ, Garcia-Garcia HM, Wykrzykowska JJ, Ligthart JMR, et al. First case of stenting of a vulnerable plaque in the SECRITT I trial - the dawn of a new era? Nat Rev Cardiol. 2009;6(5):374-8.

[27] Lebeau R, Serri K, Morice M, Hovasse T, Unterseeh T et al. Assessment of left ventricular ejection fraction using the wall motion score index in cardiac magnetic resonance imaging. Arch Cardiovasc Dis. 2012 Feb;105(2):91-8.

[28] Hasan M, Rubenstein D, Yin W. Effects of cyclic motion on coronary blood flow. J Biomech Eng. 2013 Oct 4;135(12). 